\title{
Ultrasound Guided Regional Anesthesia
}

\author{
Stuart A. Grant, David B. Auyong. Oxford University Press, New York, NY, United States \\ of America, 2017, Second Edition - \$121 CDN. Number of pages: 268. ISBN: 978-0-19- \\ 023180-4
}

\author{
Kim Wild, MBBS, FRCA · Vincent Chan, MD, FRCPC, FRCA
}

Received: 20 April 2017/ Accepted: 11 May 2017/Published online: 2 June 2017

(C) Canadian Anesthesiologists' Society 2017

If you plan to purchase a textbook on the practice of regional anesthesia, there are two issues to consider. First, you should select a text that is current and comprehensive, inclusive of established novel techniques. Second, you need to choose between a pocket guide, a heavyweight tome, or an intermediate-sized book-the latter option is arguably the most useful. In the clinical environment, pocket guides often contain too little information, and full reference texts contain too much detail for day-to-day use. In the preface of this intermediate-sized book, Grant and Auyong detail their goal- "a step-by-step practical companion...[that] should be used as an everyday working practice guide to cover the most common blocks and surgical procedures." Consequently, their intended audience encompasses both the novice learner and the experienced regional practitioner.

This second edition incorporates several important changes, including refined images, popular novel techniques, and a new appendix entitled "What Block for What Surgery?"

The text is organized into four chapters with emphasis on basic principles, upper limb, lower limb, and trunk and spine blocks. Each of these chapters is subdivided into multiple topics. Chapter 1, "Basic Principles of Ultrasound Guided Nerve Block", details the "nuts-and-bolts" information on key subjects such as physics, machine settings, technique, and ergonomics, to name a few. The text is written in a very practical format that reads like a "how-to-do-it" tutorial and takes the practitioner through the entire block procedure-from optimizing machine

K. Wild, MBBS, FRCA $(\bowtie) \cdot$ V. Chan, MD, FRCPC, FRCA

Toronto Western Hospital, Toronto, ON, Canada

e-mail: kimwild@gmail.com settings, to visualizing the nerve, needling technique, and position ergonomics.

Additionally, the chapter is full of practical advice on "how-to-do-it-well", and there is a useful section that covers key strategies for success. The new appendix for this second edition serves as a quick reference guide for choosing the optimal nerve block technique. While the recommendations are useful, they should be considered in conjunction with your local hospital protocols, as some of the suggested methods might result in prolonged motor block, contrary to the principles ensconced in most enhanced recovery programs.

Chapter 2 "Upper Limb Ultrasound Guided Regional Anesthesia", Chapter 3 "Lower Limb Ultrasound Guided Nerve Block", and Chapter 4 "Trunk and Spine Ultrasound Guided Regional Anesthesia" cover a plethora of procedures that will satisfy the majority of regional anesthetists. Beyond the established common techniques, such as brachial plexus blockade, multiple novel blocks are described in detail, including fascial plane techniques, for example, the serratus plane, pectoral nerve, quadratus lumborum, and suprainguinal fascia iliaca blocks. Each regional technique is logically organized, with sections covering clinical anatomy, clinical applications, performance, alternate approaches (e.g., out-of-plane vs in-plane techniques or the three different approaches for the lumbar plexus block), catheter techniques, and clinical pearls.

So why choose this book over other textbooks concerned with ultrasound-guided regional anesthesia? This textbook has several distinguishing features. The illustrations are superb; the ultrasound images for each procedure are shown in duplicate-an unaltered raw ultrasound image is juxtaposed with a clearly labelled 
coloured overlay-providing excellent clarity. The use of abbreviations is kept to a minimum-i.e., each structure is nearly always presented with its full name, removing the need to translate confusing codes. Positioning of the patient, ultrasound machine, and needle are dealt with by simple labelled photographs. The directions on how to perform the blocks are detailed, easy to follow, and peppered with useful tips. If there is a popular alternative approach, the authors describe that as well.

There are, however, a few limitations which are customary in most books of this size. Sections on anatomy are brief, but the authors advise the common mantra-i.e., that an anatomy textbook is your best companion to a regional anesthesia textbook. Detailed descriptions of less commonly performed approaches to nerve blocks are not always included. In addition, the focus of this textbook is on single-shot techniques; consequently, sections on continuous catheter techniques are not equally detailed and often refer to a generic description of catheter placement covered in the initial chapter.

In summary, this textbook provides detailed yet succinct instructions for an extensive range of techniques, including a number of novel blocks. It is written as a guide for the practising clinician and therefore is very accessible. It also features a multitude of clinical pearls that will aid both novice and experienced practitioners alike. For an up-todate practical reference on this subject matter, we highly recommend adding Grant and Auyong's textbook to your short list. It should certainly provide useful clinical guidance for many years to come.

Conflicts of interest None declared.

Editorial responsibility This submission was handled by Dr. Steven Backman, Associate Editor, Canadian Journal of Anesthesia. 